\title{
The ALICE electromagnetic calorimeter EMCAL: its status and its physics capabilities
}

\author{
Alessandra Fantoni ${ }^{1}$ \\ Laboratori Nazionali di Frascati dell'INFN \\ Via E. Fermi 40, 00044 Frascati (Roma), Italy \\ E-mail: alessandra.fantoni@lnf .infn.it
}

\begin{abstract}
ALICE (A Large Ion Collider Experiment) at LHC is designed to carry out comprehensive measurements of high energy nucleus-nucleus collisions, in order to study QCD matter under extreme conditions and to explore the phase transition between confined matter and the QuarkGluon Plasma. The Electromagnetic Calorimeter (EMCAL), fully installed in January 2011, significantly enhances ALICE's capabilities for jet measurements. In this contribution a brief description of the EMCAL with its characteristics will be given, together with the status of EMCAL commissioning and performance. Moreover, first results from data taking will be presented and an extension of EMCAL for jet-jet and $\gamma$-jet physics will be discussed.
\end{abstract}

Workshop on Discovery Physics at the LHC-KRUGER2010

Kruger National Park, Mpumalanga, South Africa

December 05 - 10, 2010

\footnotetext{
1 for the ALICE Collaboration
} 


\section{Introduction}

ALICE (A Large Ion Collider Experiment) at the LHC is designed to carry out comprehensive measurements of high energy nucleus-nucleus collisions, in order to study QCD matter under extreme conditions and to explore the phase transition between confined matter and the Quark-Gluon Plasma (QGP) [1,2].

ALICE contains a wide array of detector systems for measuring hadrons, leptons and photons. The ALICE detector is described in detail in [3]. The Electromagnetic Calorimeter (EMCAL), fully installed in January 2011, significantly enhances ALICE's capabilities for jet measurements. EMCAL is designed to provide the following functions: efficient and unbiased fast level L0 and L1 trigger on high energy jets; measurement of the neutral portion of jet energy; improvement of jet energy resolution; measurement of high momentum photons, $\pi^{0}$ and electrons; $\gamma / \pi^{0}$ discrimination up to $30 \mathrm{GeV}$ (using invariant mass and shower shape techniques only); electron/hadron separation for momenta larger than $10 \mathrm{GeV} / \mathrm{c}$; high uniformity of response for isolated electromagnetic clusters.

This paper presents a description of the EMCAL, its characteristics, its present status, and its first results. In addition an EMCAL upgrade which would expand its acceptance for di-jet and hadron-jet correlation measurements is discussed.

\section{EMCAL description and characteristics}

The overall design of the EMCAL is heavily influenced by its integration within the ALICE [3] setup, which constrains the detector acceptance to a region of about $110^{\circ}$ in the azimuthal angle $\phi,-0.7 \leq \eta \leq 0.7$ in pseudo-rapidity and $4.35 \mathrm{~m}<\mathrm{R}_{\mathrm{EMCAL}}<4.7 \mathrm{~m}$ radial distance. The chosen technology is a layered Lead $(\mathrm{Pb})$-Scintillator (Scint) sampling calorimeter with wavelength shifting (WLS) fibers that run longitudinally through the $\mathrm{Pb} / \mathrm{Scint}$ stack providing the light collection (Shashlik) [4]. The basic building block is a module consisting of $2 \times 2$ optically isolated towers which are read out individually. All modules in the calorimeter are mechanically and dimensionally identical. Each module has a rectangular cross section in the $\phi$ direction and a trapezoidal cross section in $\eta$ with a full taper of $1.5^{\circ}$, and spans $\Delta \eta \times \Delta \phi=$ $0.014 \times 0.014$. A scheme of the module is shown in Fig. 1 , together with the dimensions in $\mathrm{mm}$. White, acid free, bond paper serves as a diffuse reflector on the scintillator surfaces and provides friction between layers. The scintillator edges are treated with $\mathrm{TiO}_{2}$ loaded reflector to improve the transverse optical uniformity within a single tower and to provide tower to tower optical isolation better than $99 \%$. The requirement of a compact detector consistent with the EMCAL integration volume and the chosen detector thickness of about 20 radiation lengths, results in a lead to scintillator ratio by volume of about 1:1.22 corresponding to a sampling geometry of $\mathrm{Pb}(1.44 \mathrm{~mm}) / \mathrm{Scint}(1.76 \mathrm{~mm})$. The physical characteristics of the modules are summarized in Table 1.

Scintillation photons produced in each tower are captured by an array of 36 Kuraray Y-11 (200 M), double clad, wavelength shifting (WLS) fibers. Each fiber within a given tower terminates in an aluminized mirror at the front face of the module and is integrated into a polished, circular group of 36 fibers at the photo sensor end at the back of the module.

The $6.8 \mathrm{~mm}$ diameter fiber bundle from a given tower connects to the Avalanche Photodiode (APD) through a short light guide/diffuser. The selected photo sensor is the Hamamatsu S866455 avalanche photodiode chosen for operation in the high field inside the ALICE magnet. The APDs are operated at moderate gain for low noise and high gain stability in order to maximize energy and timing resolution. 
The number of primary electrons generated in the APD by an electromagnetic shower is $\sim 4.4$ electrons $/ \mathrm{MeV}$. The reverse bias voltage of the APDs are individually controlled to provide an electron multiplication factor (M) of 30 resulting in a charge output of $\sim 132$ electrons/ $\mathrm{MeV}$ from the APDs. All APDs used have been previously calibrated [5]. The charge output from the APD is integrated by a Charge Sensitive Preamplifier (CSP) with a short rise time of $\sim 10 \mathrm{~ns}$ and a long decay time of $\sim 130 \mu$ s, i.e., approximately a step pulse. The readout electronics is similar to that of the Photon Spectrometer (PHOS): a detailed description is given in [6].

Twelve modules are assembled with a structural strongback to form a strip-module, a selfsupporting unit like the module. A collection of 24 strip-modules forms a super-module and the full EMCAL is formed by ten super-modules. More details on the mechanical assembly can be found in [7].

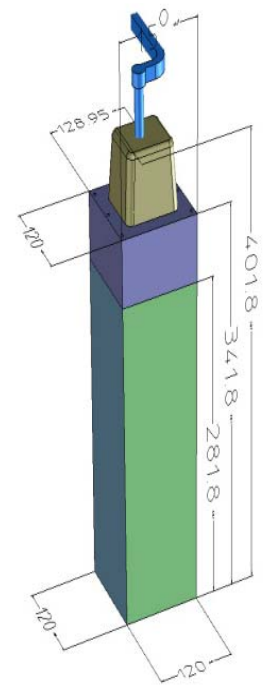

\begin{tabular}{|l|l|}
\hline Parameter & Value \\
\hline Tower size (at $\eta=0)$ & $\sim 6.0 \times \sim 6.0 \times 24.6 \mathrm{~cm}^{3}$ \\
Tower size & $\Delta \phi \times \Delta \eta=0.0143 \times 0.0143$ \\
Sampling ratio & $1.44 \mathrm{~mm} \mathrm{~Pb} / 1.76 \mathrm{~mm}$ Scint. \\
Layers & 77 \\
Scintillator & Polystyrene (BASF143E+ \\
& $1.5 \% \mathrm{pTP}+0.04 \%$ POPOP) \\
Absorber & Natural lead \\
Effective RL $\mathrm{X}_{0}$ & $12.3 \mathrm{~mm}$ \\
Effective MR $\mathrm{R}_{\mathrm{M}}$ & $3.20 \mathrm{~mm}$ \\
Effective density & $5.68 \mathrm{~g} / \mathrm{cm}^{3}$ \\
Sampling fraction & $1 / 10.5$ \\
Radiation length $\left(\mathrm{RL} \mathrm{X}_{0}\right)$ & 20.1 \\
\hline
\end{tabular}

Fig.1 (left): A single EMCAL module with the dimension in $\mathrm{mm}$. Table 1 (right): EMCAL module physical parameters. Here, RL stands for Radiation Length and MR for the Moliere Radius.

\section{Results from test beams}

The performance of a $4 \times 4$ array of prototype modules and of a $4 \times 4$ array of final design modules for the ALICE EMCAL has been studied in test beam measurements at FNAL (2005) and CERN (2007), respectively. All towers were instrumented with the full electronics chain with shapers and APD gains operated as planned in ALICE. A LED calibration system was installed in order to monitor time-dependent gain changes. The readout of the front end electronics used the standard ALICE data acquisition system.

The final results are fully described in details in [8], showing an energy resolution of $\sigma(E) / E=$ $(1.7 \pm 0.3)+(11.1 \pm 0.4) / 2+(5.1 \pm 0.3) / \mathrm{E}$ (where $\mathrm{E}$ is in $\mathrm{GeV}$ ), in agreement with the design criteria, as for the full performance. 


\section{The EMCAL status}

The assembly of EMCAL modules started at the end of 2008. In 2009 four supermodules have been installed in ALICE and in 2010 they have been operational and taking data. The corresponding angular coverage is $\Delta \eta \times \Delta \phi=1.4 \times 1.05$. Extensive commissioning of the readout electronics, L0, L1 and HLT has been carried forward together with APD bias optimization and gain monitoring. First physics events have been successfully reconstructed. The modules assembly has been completed in summer 2010: at the time of the Workshop, all six super-modules have been assembled, tested, calibrated and checked again in order to confirm their full operationality before the insertion in ALICE, scheduled in January 2011.

\section{The EMCAL performance and its physics capabilites}

After months of commissioning and running with cosmic rays, at the end of 2009 it was possible to see the response of EMCAL with the first proton-proton collisions at the center of mass energy $\sqrt{s}=900 \mathrm{GeV}$ and in 2010 at $\sqrt{s}=7 \mathrm{TeV}$. In the early commissioning phase of the LHC, the first events were analyzed and displayed in the counting room using the reconstruction software implemented in the high Level Trigger (HLT) to analyze the events in real time, marking the start of the physics exploitation of the ALICE experiment. First results have been immediately obtained and published [9]. The four EMCAL super-modules have been continuously present in the data taking. A picture of them is shown in Fig.2 (right panel), where only the two in front are visible.

The $\gamma \gamma$ invariant mass spectra for $\pi^{0}$ and $\eta$ in pp collisions at $900 \mathrm{GeV}$ and $7 \mathrm{TeV}$ have been obtained and used to prove the performance of the EMCAL: both spectra at the latest energy are shown in Fig.3. These measurements provide also reference spectra for heavy ion collisions at LHC.

Heavy ion collisions at LHC have been produced in November 2010 at the center of mass energy per nucleon pair of $2.76 \mathrm{TeV}$. EMCAL was able to run with the rest of ALICE, despite being the last detector (partially) installed. A picture of a $\mathrm{Pb}-\mathrm{Pb}$ collision from the HLT display is shown on the left panel of Fig.4, where the signals in EMCAL are clearly visible.
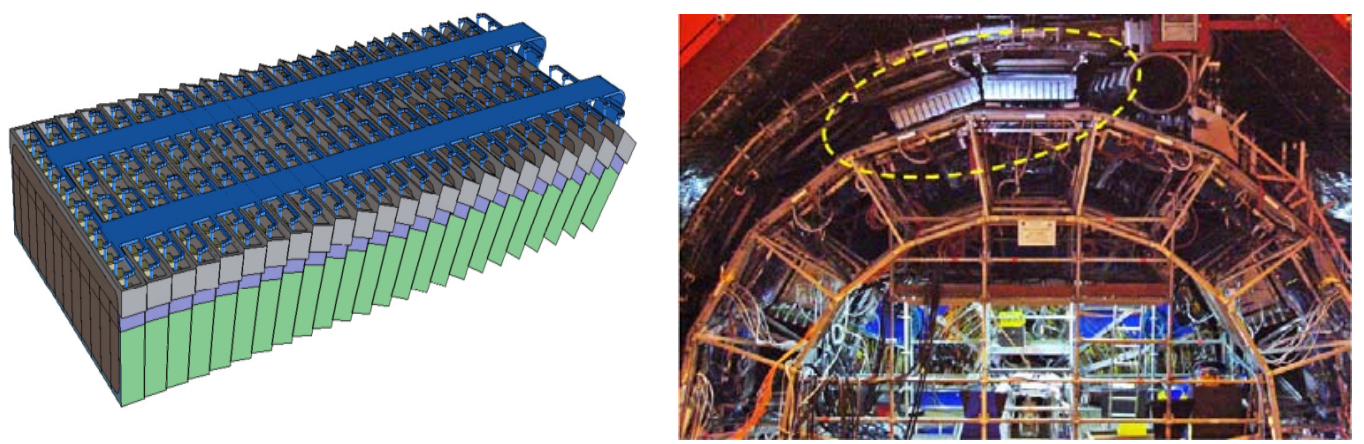

Fig.2 Left panel: a super-module formed by 24 strip-modules. Right panel: four super-modules installed, only those two in front are visible.

EMCAL will complete ALICE's capabilities to measure jet quenching, by providing an efficient and unbiased fast jet trigger (Level 0/1) and measuring of the neutral fraction of the jet energy. Usually the jet reconstruction in elementary collisions is done using electromagnetic and hadronic calorimeters, while in ALICE the hadronic energy is measured using high resolution 
charged particle tracking and the electromagnetic energy is measured using the EMCAL. Charged jets in ALICE are reconstructed using a variety of different algorithms, with different sensitivities to background and different subtraction schemes, as reported on the right panel of Fig.4, where a good agreement between all jet finders is found.
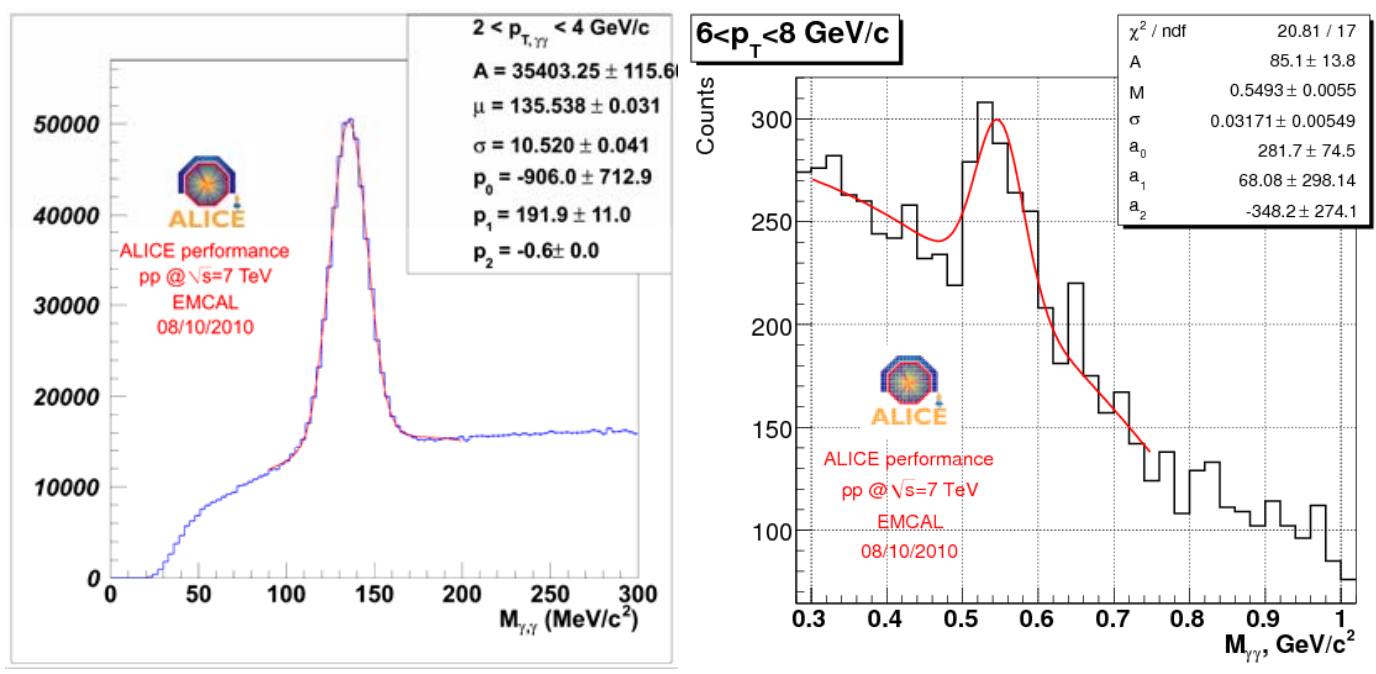

Fig. $3 \pi^{0}$ (left panel) and $\eta$ (right panel) $\gamma \gamma$ invariant mass spectra from $180 \mathrm{M}$ events in pp collisions at $7 \mathrm{TeV}$ in a selected transverse momentum bin. For the cluster selection, the number of cells in a cluster is 2 , while the minimum energy of the cluster is $0.5(0.3) \mathrm{GeV}$ for $\pi^{0}(\eta)$.
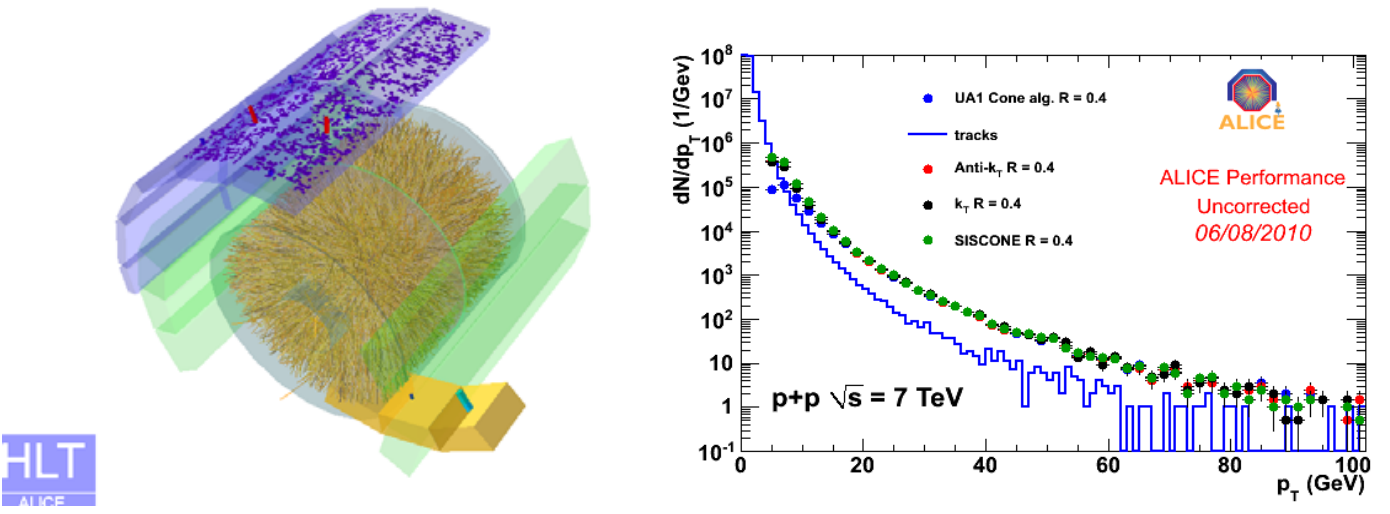

Fig.4 Left panel: event from HLT display during Pb-Pb collisions. Right panel: charged jet reconstructed in ALICE with different algorithms.

\section{The EMCAL upgrade: DCAL}

Even though EMCAL was the last ALICE detector to be proposed, approved, assembled, and it is still only partially installed, the first upgrade approved by the ALICE collaboration (November 2009) is an extension of EMCAL, the Di-jet Calorimeter (DCAL) [10]. The DCAL expands the physics capabilities of the EMCAL by enabling back-to-back 
correlation measurements, which are impossible with the EMCAL alone, but are essential to obtain a complete picture of the physics addressed by the EMCAL. Together, the DCAL and EMCAL form a two-arm electromagnetic calorimeter. The EMCAL subtends $110^{\circ}$ and the DCAL subtends $60^{\circ}$ in $\phi$, with both detectors covering $|\eta|<0.7$, thereby providing good acceptance for di-jets with radii $\mathrm{R} \leq 0.4$ up to transverse momenta $\mathrm{p}_{\mathrm{T}} \sim 150 \mathrm{GeV} / \mathrm{c}$. Simulation studies of the DCAL have been carried out and have verified that the technology originally developed for and implemented in the EMCAL meets all the needs of the DCAL project. As a consequence, from a technical perspective, DCAL is an extension of EMCAL. DCAL supermodules are built exactly as they are in EMCAL, out of strip-modules, but with reduced length in $\eta$ : infact, each DCAL strip-module contains 16 modules instead of 24 present in EMCAL.

DCAL will be situated immediately adjacent to PHOS on both the ALICE A and C sides, causing a small gap in $\eta(\delta \eta \sim 0.02)$ between the sensitive volumes of the two detectors, due to the super-module structure. DCAL+PHOS can be considered as one integrated detector system for the study of jets, consequently all simulations done include PHOS as well as DCAL supermodules.

The assembly of the DCAL modules started in summer 2010, after completion of EMCAL. More than 300 modules have been assembled, that is more than 1.5 DCAL super-module. The assembly is expected to be completed in spring 2011, in order to be installed for 2012 runs.

On the left panel of Fig.5 is shown a schematic view of the 6 DCAL super-modules with the PHOS super-modules in between and on the right panel the beam view of EMCAL and DCAL is illustrated.
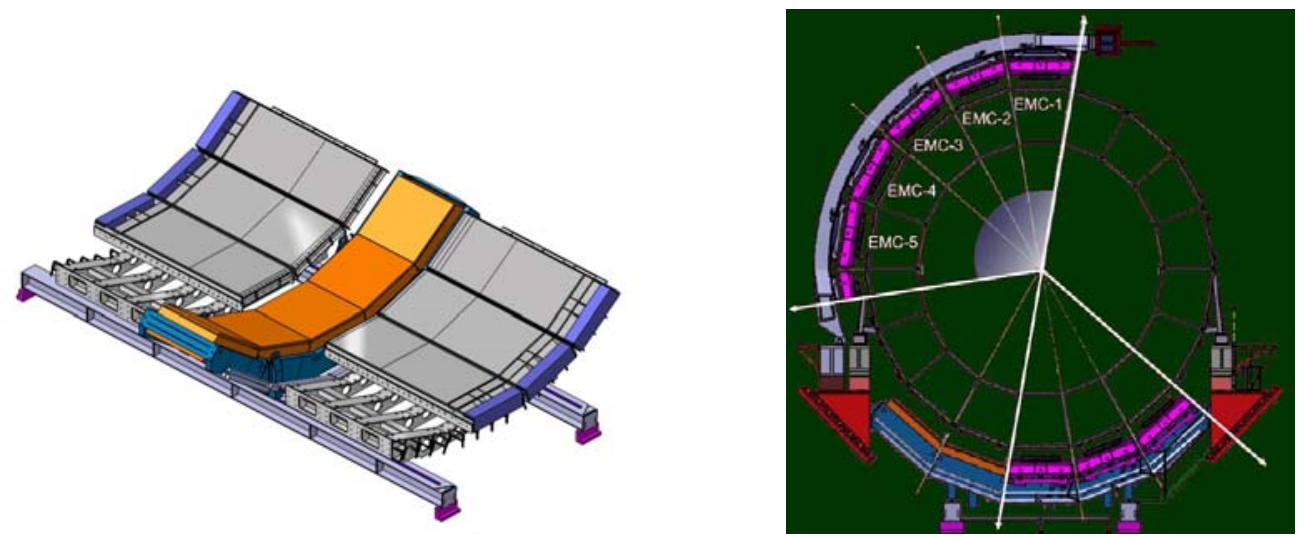

Fig.5: Left panel: 6 DCAL super-modules (in gray) with the PHOS super-modules (in orange) in between. Right panel: beam view of EMCAL and DCAL.

\section{Conclusions}

An accurate description of the ALICE electromagnetic calorimeter, with its characteristics has been given. Final results from measurements done in test beams in 2005 and 2007 have been reported, demonstrating that the performances of EMCAL satisfy all the design criteria. The status of the assembly and installation of EMCAL is illustrated: four supermodules are installed in ALICE for 2009 and 2010 data taking.

The assembly of EMCAL modules has been completed in summer 2010 and the assembling/checking/calibration of the six corresponding super-modules has been concluded in December, to be ready for the full installation during the winter shutdown in January 2011. 
An upgrade for EMCAL, denominated DCAL for di-jet and hadron-jet physics, is illustrated: the assembly of the modules started in summer 2010 and will be completed in spring 2011, ready for the installation for the next shutdown.

\section{References}

[1] F. Carminati et al. (ALICE Collaboration), J. Phys. G30 1517 (2004).

[2] ALICE Collaboration, Physics Performace Report, Vol 2, J. Phys. G (2006).

[3] K. Aamodt et al. (ALICE Collaboration), JINST 3 S08002 (2008).

[4] ALICE Collaboration, ALICE Technical Design Report Electromagnetic Calorimeter, CERN LHCC-2008-014, 1 December 2008.

[5] A. Badalà et al., Nucl. Instrum. Methods A610, 200 (2009).

[6] PHOS Collaboration, ALICE Technical Design Report, CERN/LHCC 99-4, 5 (1999).

[7] F. Ronchetti et al., J. Phys. Conf. Series 160, 012012 (2009).

[8] J. Allen et al., Nucl. Instrum. Methods A615, 6 (2010).

[9] K. Aamodt et al. (ALICE Collaboration), Eur. Phys. J. C65, 111 (2010); Phys.Rev.Lett.105, 252301 (2010); Phys.Lett.B693， 53 (2010); Phys.Rev.D82, 052001 (2010); Phys.Rev.Lett.105, 072002 (2010); Eur.Phys.J.C68, 345 (2010); Eur.Phys.J.C68, 89 (2010).

[10] J. Allen et al. (ALICE Collaboration), ALICE DCal: An addendum to the EMCAL Technical Design Report, CERN LHCC-2010-011, 20 June 2010. 